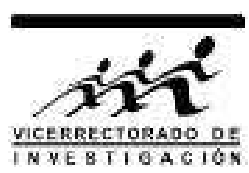

\title{
Cálculo de la dosis absorbida en el plano medio aplicando el método de dosimetría in vivo para el caso de la irradiación de cuerpo total
}

\author{
F. Ubillús ${ }^{1}$, J. Márquez ${ }^{1,2}$ y C. Herrera ${ }^{2}$ \\ ${ }^{1}$ Facultad de Ciencias Físicas, Universidad Nacional Mayor de San Marcos, Ap. Postal 14-0149, Lima, Perú \\ ${ }^{2}$ Instituto Nacional de Enfermedades Neoplásicas, Surquillo, Lima, Perú
}

Recibido 4 setiembre 2013 - Aceptado 10 octubre 2013

La Irradiación de Cuerpo Total (ICT) con haces de fotones es una técnica utilizada en el tratamiento de enfermedades hematopoyéticas, a fin de lograr las condiciones de esterilidad necesarias para realizar trasplantes de médula ósea. Para esto se debe prescribir la dosis en el plano medio del paciente y tener una distribución aproximadamente exacta de la dosis (desviación del $\pm 5 \%$ de la dosis prescrita) en todo el cuerpo, pero debido a la geometría irregular del paciente no es posible utilizar sistemas de planificación de tratamiento; es por esto que se recurre a la dosimetría in vivo.

La aplicación del método de dosimetría in vivo consiste en un algoritmo de cálculo basado en los valores de dosis de entrada y salida medidos por los detectores de Luminiscencia Ópticamente Estimulada OSL $\left(\mathrm{Al}_{2} \mathrm{O}_{3}: \mathrm{C}\right)$ ubicados en las superficies de un fantoma homogéno, el cual es irradiado con haces de fotones de $6 \mathrm{MV}$ generados por el Acelerador Lineal Siemens Primus Mid (Mevatron). Posteriormente estos resultados son comparados con las lecturas de dosis tomadas por una cámara de ionización (PTW Freiburg Modelo TW31010-2167) ubicada en plano medio. De igual forma se procede para el caso en el que el haz es atenuado en un $22.72 \%$, empleando una protección de plomo de $4 \mathrm{~mm}$ (utilizado como protección de órganos críticos), comprobándose desviaciones menores al $\pm 10 \%$ en ambos casos. Por tanto, el uso de estos detectores permite desarrollar un método dosimétrico alternativo, que contribuye a garantizar la calidad del tratamiento.

Palabras claves: Irradiación de cuerpo total, dosímetría in vivo, dosimtría OSL.

\section{Middle plane absorbed dose calculation applying in vivo dosimetry during total body irradiation}

Total Body Irradiation (ICT) with photon beams is a technique used in the treatment of haematopoietic diseases in order to achieve sterile conditions necessary to perform bone marrow transplants. For this dose should be prescribed in the plane of the patient and have a roughly accurate distribution of the dose $( \pm 5 \%$ deviation from the prescribed dose) throughout the body, but due to the irregular geometry of the patient can not use planning systems treatment, which is why it resorts to in vivo dosimetry.

The application of the method of dosimetry in vivo is an algorithm based on the values of entry and exit doses measured by the detectors optically stimulated luminescence OSL $\left(\mathrm{Al}_{2} \mathrm{O}_{3}: \mathrm{C}\right)$ located on the surface of a phantom uniformity, which is irradiated with beams of $6 \mathrm{MV}$ photons generated by the Siemens Primus Linear Accelerator Mid (Mevatron). Subsequently these results are compared with the dose readings taken by an ionization chamber (PTW-Freiburg TW31010 Model 2167) located in the middle plane. The same shall be for the case where the beam is attenuated by $22.72 \%$, using a lead shielding of $4 \mathrm{~mm}$ (used as protection of critical organs), checking deviations were less than $\pm 10 \%$ in both cases. Therefore, the use of these detectors can develop an alternative dosimetric approach, which helps ensure the quality of treatment. Keywords: Total body irradiation, in vivo dosimetry, OSL dosimetry.

La Irradiación de Cuerpo Total, ICT, con haces de fotones de $6 \mathrm{MV}$, es una técnica especial de radioterapia en la cual se entrega a todo el cuerpo del paciente una dosis uniforme con una exactitud de -10 a $+5 \%$ de la dosis prescrita, que suele ser utilizada como referencia al aplicar la técnica de ICT, según el protocolo AAPM Report № 17, 1986 [2], a excepción de algunos órganos específicos, que son parcial o totalmente protegidos [1] 2 . 
En este trabajo se plantea validar la aplicación del método de dosimetría in vivo para el caso de ICT bilateral, a partir del cálculo de dosis absorbida en plano medio, empleando un algoritmo basado en las lecturas de dosis de entrada y salida de un fantoma. Para ello se debe evaluar en primer lugar la calidad del haz de $6 \mathrm{MV}$, usando valores de porcentaje de dosis en profundidad y de igual forma detrás de un bloque de transmisión parcial, usado como protección de órganos en riesgo luego de evaluar el porcentaje de atenuación correspondiente al espesor de plomo requerido como protección, tal que permita atenuar parcialmente la intensidad del haz hasta en un $20 \%$, valor que es recomendable para el caso de protección de órganos críticos, según el Protocolo AAPM Report № 17 , 1986 [2], aproximadamente.

Luego de realizar los respectivos cálculos de las dosis se deben comparar con el valor medido por la cámara de ionización ubicada en esa posición, a fin de obtener desviaciones que no superen el $\pm 10 \%$ y de esta manera comprobar si el método puede ser llevado a casos rutinarios de ICT, a partir de los resultados y porcentajes de desviación obtenidos, aplicando las mismas condiciones y parámetros; tal que el uso de estos detectores permita desarrollar un método dosimétrrico alternativo; así como contribuir a garantizar la calidad del tratamiento [1,3

\section{Materiales y métodos}

El equipamiento utilizado consistió de un Acelerador Lineal Siemens Primus Mid (Mevatron), con el que se irradió un fantoma homogéneo compuesto de láminas de acrílico $\left(\rho=1.19 \mathrm{~g} / \mathrm{cm}^{2}\right)$, en el cual se colocaron los dosímetros OSL $\left(\mathrm{Al}_{2} \mathrm{O}_{3}: \mathrm{C}\right)$ que fueron posteriormente leídos por medio de un sistema dosimétrico llamado Lector MicroStar. Para la comprobación se utilizó, una Cámara de Ionización, Cl, (PTW Freiburg Modelo TW31010-2167) cuya carga colectada es leída por un Electrómetro. Para el caso en el que se necesitó atenuar parcialmente el haz se utilizó un disco de plomo (espesor $=4 \mathrm{~mm}$ ).

\section{Distribución de dosis a lo largo del eje central}

Para la evaluación de la calidad del haz es necesario analizar el comportamiento de la curva "PDP vs d". El PDP deriva generalmente de la relación entre la lectura para una profundidad donde la dosis es máxima $d_{m}$ ( Tabla 1) y la lectura en cualquiera de las profundidades $d$, medida por la $\mathrm{Cl}$ en $\mathrm{nC}$ [4,5].

Para llevar a cabo este procedimiento, la $\mathrm{Cl}$ debe ser colocada en el centro geométrico del fantoma detrás de un espesor mínimo $(0.8 \mathrm{~cm})$ y coincidir con el eje central del haz (Figura 1), tal que después de cada irradiación se agregue un espesor determinado delante de la $\mathrm{Cl}$, manteniendo siempre un tamaño de campo, $T C=10 \times 10 \mathrm{~cm}^{2}$. El tamaño de campo $T C=10 \times 10 \mathrm{~cm}^{2}$ es elegido para una distancia de la fuente-isocentro, $D F I=100 \mathrm{~cm}$, para ser proyectado a una distancia fuente-superficie, $D F S=300$ $\mathrm{cm}$ con $30 \times 30 \mathrm{~cm}^{2}$ [4] y un tiempo $(U M=100) \mathrm{fi}-$ jo [4-6].

Tabla 1: Profundidades típicas $d_{m}$ donde la dosis es máxima, para haces de fotones de distintas energías [4].

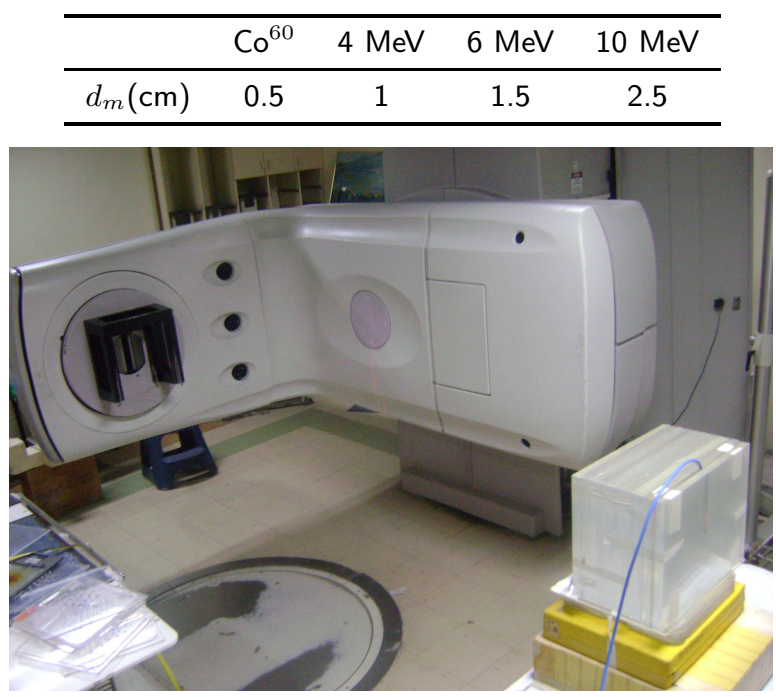

Figura 1: Arreglo experimental utilizado para obtener las curvas de $P D P$ y valores de dosis a lo largo del eje central del haz.

\section{Evaluación del espesor de plomo requerido como protección}

Para determinar el espesor de plomo necesario para atenuar parcialmente el haz de radiación, hasta en un $20 \%$; se requiere de una $\mathrm{Cl}$ ubicada a una $D F S$ fija y de pequeños discos de plomo de diferentes espesores, que van a ser ubicados a la salida del colimador. Para este caso se requiere que delante de la cámara se coloque un espesor mínimo de acrílico tal como se muestra en la Figura 2. Se realizará este procedimiento las veces que sea necesario hasta obtener el porcentaje de atenuación requerido, relacionado a las lecturas $(\mathrm{nC})$ de la $\mathrm{Cl}$ [2].

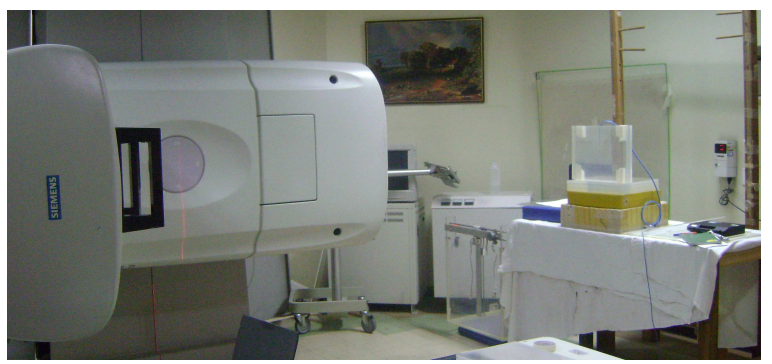

Figura 2: Arreglo experimental utilizado para evaluar el espesor de plomo requerido como protección. 


\section{Algoritmo de cálculo de dosis en el plano medio}

La dosis en plano medio $\left(D_{d / 2}\right.$ y $\left.D_{d / 2}^{\prime}\right)$ se calcula tomando la media aritmética de la dosis de entrada y salida $\left(D_{e}, D_{s} \circ D_{e}^{\prime}, D_{s}^{\prime}\right)$, donde $D_{e}^{\prime}, D_{s}^{\prime}$ y $D_{d / 2}^{\prime}$ son los valores de las dosis medidas detrás de la protección plomada, bajo las mismas condiciones dosimétricas [1], corregida por un factor de corrección, $F C$, mostrado en

$$
D_{d / 2}=\frac{D_{e}+D_{s}}{2} \times F C
$$

que a su vez se expresa como

$$
F C=\frac{2 \times P D P(d / 2)}{P D P\left(d_{m}\right)+P D P\left(d-d_{m}\right)},
$$

donde PDP corresponde al porcentaje de la dosis en profundidad medida en condiciones de ICT usando la $\mathrm{Cl}$. Por definición $\operatorname{PDP}\left(d_{m}\right)=100$, siendo $d$ el espesor del paciente y $d / 2$ el espesor medio [1].

\section{Método para obtener valores de dosis por medio de los detectores OSL}

Los dosímetros se calibran comparando su respuesta con la dosis determinada con la $\mathrm{Cl}$ situada en el centro del campo y a la profundidad del máximo de la dosis. El factor de calibración se obtiene mediante [7, 8]

$$
F_{c a l}=\frac{D_{c i}}{L_{\text {nanodot }}}
$$

donde $D_{c i}$ es la dosis determinada por la $\mathrm{Cl}$ y $L_{\text {nanodot }}$ es la lectura del detector nanodot [7].

Tabla 2: Factores de correción y calibración utilizados para el cálculo de dosis (cGy) en la entrada y salida [7].

\begin{tabular}{cc}
\hline Parámetro & Valor \\
\hline$F_{\text {dis }}$ & 1.000 \\
$F_{\text {uso }}$ & 1.010 \\
$F_{\text {tasa }}$ & 1.020 \\
$F_{\text {ang }}$ & 1.020 \\
$F_{\text {ene }}$ & 1.020 \\
$F_{\text {cal }}$ & 1.725 \\
$F_{\mu \text { Star }}$ & 3.040 \\
\hline
\end{tabular}

Puesto que la sensibilidad de los detectores depende de la tasa de dosis, de la energía y de la temperatura, cuando las condiciones de medida difieran de las condiciones de caracterización se deberán aplicar factores de corrección (Tabla 2) para obtener la dosis a partir de la lectura del detector, tales como el factor de corrección por uso $\left(F_{\text {uso }}\right)$, por tasa de dosis $\left(F_{t a s a}\right)$, por distancia $\left(F_{d i s}\right)$, por energía $\left(F_{\text {ene }}\right)$, por ángulo de incidencia $\left(F_{a n g}\right)$ y debido al uso del lector MicroStar $\left(F_{\mu \text { Star }}\right)$ [7]. Por tanto la dosis medida por el detector está dada por

$$
D=L \cdot F_{c a l} \cdot \Pi F_{i}
$$

donde $L$ es la lectura del dosímetro OSL, $F_{c a l}$ es su factor de calibración y $\Pi F_{i}$ es el producto de todos los factores de corrección [7].

\section{Cálculo de $D_{1 / 2}$ experimental según el protocolo $\mathrm{N}^{\circ} 398$}

Para determinar la dosis en el plano medio experimental es necesario contar con las lecturas procedentes de una $\mathrm{Cl}$ previamente calibrada, bajo una calidad de referencia $Q_{o}$, 9],

$$
D_{w, Q}=M_{Q} N_{D, w, Q_{o}} k_{Q, Q_{o}},
$$

donde $M_{Q}[\mathrm{nC}]$ es la lectura de la $\mathrm{Cl}$, que incluye el producto $\Pi k_{i}$ de los factores de corrección por las magnitudes de influencia, $N_{D, w}, Q_{o}[\mathrm{~Gy} / \mathrm{nC}]$ es el factor de calibración y $k_{Q, Q_{o}}$ es el factor de corrección que tiene en cuenta la diferencia entre la calidad del haz de referencia, $Q_{o}$, y la calidad real utilizada para el tratamiento $Q$, siendo este valor cercano a 1 [8], luego

$$
M_{Q}=M_{Q^{\prime}} \Pi k_{i}
$$

\section{Corrección por magnitudes de influencia}

El factor de calibración de una $\mathrm{Cl}$ es válido sólo para las condiciones de referencia que se aplican en la calibración. Los factores de corrección, representados en el producto $\Pi k_{i}$ son los factores de presión, temperatura y humedad $\left(k_{P T}\right)$, de calibración del electrómetro $\left(k_{\text {elec }}\right)$, de efecto de polaridad $\left(k_{p o l}\right)$, de recombinación de iones $\left(k_{s}\right)$ y por último el factor relacionado con el material cuando se trabaja con uno diferente al agua como el acrílico $\left(k_{f}\right)$ (ver Tabla 3) [4].

Tabla 3: Factores de correción y calibración utilizados para el cálculo de dosis (cGy) en plano medio [9].

\begin{tabular}{cc}
\hline Parámetro & Valor \\
\hline$k_{P T}$ & 1.040 \\
$k_{\text {elec }}$ & 1 \\
$k_{\text {pol }}$ & 0.996 \\
$k_{s}$ & 1.004 \\
$k_{f}$ & 1.031 \\
\hline
\end{tabular}

Para calcular dosis en plano medio, se toma como referencia el esquema mostrado en la Figura 3, con respecto a la ubicación de los detectores y parámetros dosimétricos requeridos. 


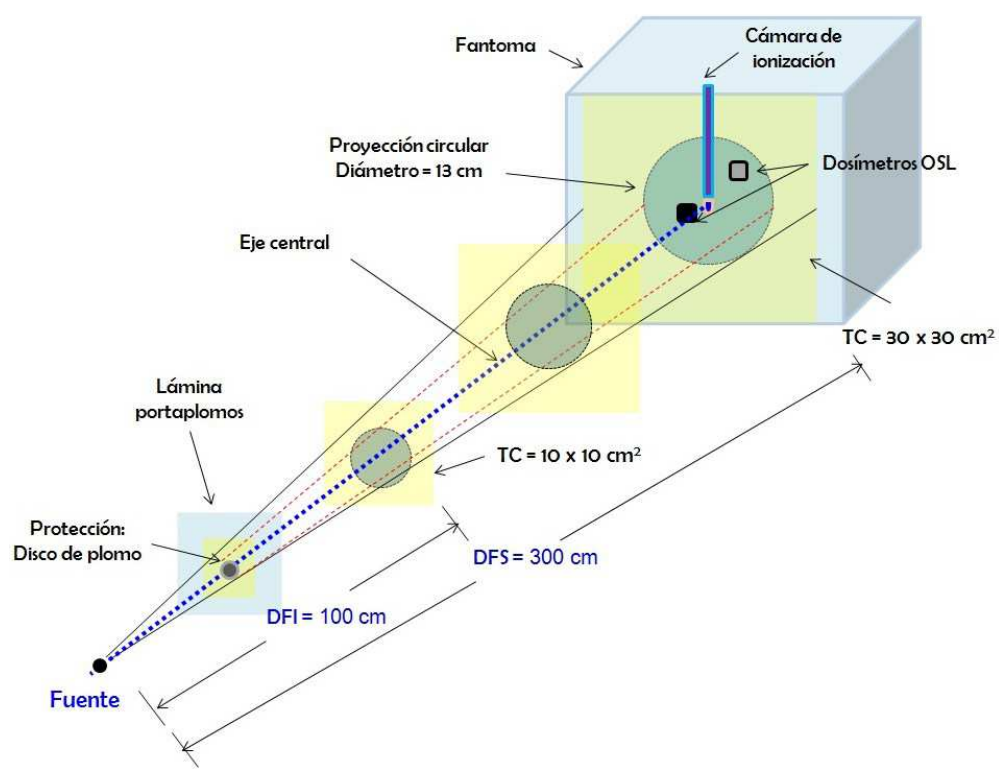

Figura 3: Ubicación de los detectores en el fantoma y los parámetros dosimétricos en condiciones de ICT.

\section{Resultados y discusiones}

Cuando evaluamos la calidad del haz por medio de los resultados de PDP (Figura 4), se observa que la dosis absorbida por el fantoma alcanzó su máximo valor entre las profundidades 1.13 y $1.57 \mathrm{~cm}$, encontrándose dentro del rango según la Tabla 1 . Esta variación se debe a que las condiciones dosimétricas difieren de las de una técnica estándar, en cuanto a las profundidades donde se ubica la $\mathrm{Cl}$, tipo de fantoma, tamaño de campo y DFS usados en casos de ICT [4,5].

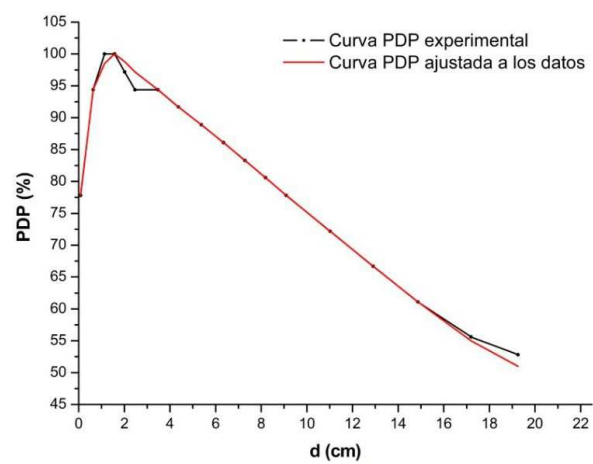

Figura 4: Curva de PDP en un fantoma homogéneo, con un $T C=10 \times 10 \mathrm{~cm}^{2}$ y a una $D F S=300 \mathrm{~cm}$, para un haz de fotones de energía $6 \mathrm{MeV}$.

Se evalúo el porcentaje de atenuación producido por dos espesores distintos (Tabla 4), lográndose una atenuación del $22.72 \%$ detrás de $4 \mathrm{~mm}$ de plomo. Las consecuencias de este exceso, generan una reducción de la tasa de dosis a lo largo del eje central, menor sensibilidad en el dosímetro y mayores efectos de dispersión [2].

Tabla 4: Evaluación del espesor de plomo requerido para atenuar el haz parcialmente.

\begin{tabular}{cccc}
\hline$N^{o}$ & $\mathrm{e}(\mathrm{mm})$ & $\mathrm{M}(\mathrm{nC})$ & Atenuación $(\%)$ \\
\hline 1 & 0 & 0.22 & 0 \\
2 & 2 & 0.19 & 13.64 \\
3 & 4 & 0.17 & 22.72 \\
\hline
\end{tabular}

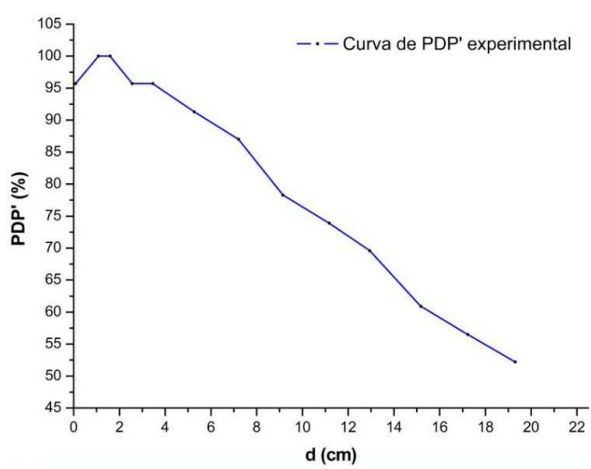

Figura 5: Curva de PDP en un fantoma homogéneo, bajo transmisión parcial; con un TC $=10 \times 10 \mathrm{~cm}^{2}$ y a una $D F S=300 \mathrm{~cm}$, para un haz de fotones de energía $6 \mathrm{MeV}$.

Cuando se evaluó la calidad del haz bajo transmisión parcial aplicando los resultados de PDP (Figura 5), se observa que entre las profundidades 1.09 y $1.59 \mathrm{~cm}$, la dosis absorbida por el fantoma alcanzó su máximo valor, encontrándose dentro del rango según la Tabla 1, debido principalmente a que la protección de plomo colocada delante del fantoma, produce mayores efectos de dispersión, 
por consiguiente esto genera una disminución de la tasa de dosis y un cambio en la calidad del haz de radiación. Otros posibles efectos que podrían también considerarse es la influencia de la divergencia del haz a mayores profundidades, la atenuación generada por el fantoma y los espesores asignados entre cada irradiación [1].

En la Tabla 5 se muestra la comparación entre los valores $D_{d / 2}$ calculado y el medido por la $\mathrm{Cl}$ así como el respectivo porcentaje de desviación. Al comparar entre este valor y el tomado por la $\mathrm{Cl}$, las desviaciones obtenidas alcanzan un máximo de $4.58 \%$, encontrándose dentro del $\pm 5 \%$ establecido [1,2].

Tabla 5: Comparación entre valores de dosis en plano medio teórico y experimental.

\begin{tabular}{ccccc}
\hline$N^{o}$ & $\begin{array}{c}\mathrm{d} \\
(\mathrm{cm})\end{array}$ & $\begin{array}{c}D_{d / 2(\text { alg })} \\
(\mathrm{cGy})\end{array}$ & $\begin{array}{c}D_{d / 2(C . I)} \\
(\mathrm{cGy})\end{array}$ & $\begin{array}{c}\text { Desviación } \\
(\%)\end{array}$ \\
\hline 1 & 6.70 & 9.66 & 10.08 & -4.31 \\
2 & 8.50 & 9.55 & 9.77 & -2.31 \\
3 & 10.37 & 9.34 & 9.77 & -4.58 \\
4 & 12.31 & 9.28 & 9.46 & -1.95 \\
5 & 16.24 & 9.01 & 9.16 & -1.69 \\
6 & 20.02 & 8.30 & 8.55 & -3.04 \\
7 & 22.80 & 7.60 & 7.94 & -4.42 \\
\hline
\end{tabular}

Se puede conseguir un porcentaje de desviación reducido si se inicia el procedimiento de dosimetría in vivo calibrando correctamente los dosímetros en la entrada y salida del haz en condiciones de ICT, ya que si se utiliza el factor de calibración correspondiente al dosímetro que está en la entrada al dosímetro colocado en la salida del haz, implica una sobreestimación de la dosis [2].

Tabla 6: Comparación de las dosis en plano medio, teórica y experimental bajo transmisión parcial del haz.

\begin{tabular}{ccccc}
\hline$N^{o}$ & $\begin{array}{c}\mathrm{d} \\
(\mathrm{cm})\end{array}$ & $\begin{array}{c}D_{d / 2}^{\prime}(\mathrm{alg}) \\
(\mathrm{cGy})\end{array}$ & $\begin{array}{c}D_{d / 2}^{\prime}(\text { C.I }) \\
(\mathrm{cGy})\end{array}$ & $\begin{array}{c}\text { Desviación } \\
(\%)\end{array}$ \\
\hline 1 & 6.70 & 8.18 & 7.94 & +2.92 \\
2 & 8.50 & 7.82 & 7.94 & -1.53 \\
3 & 10.37 & 7.35 & 7.63 & -3.82 \\
4 & 12.31 & 7.36 & 7.63 & -3.69 \\
5 & 16.24 & 6.74 & 7.33 & -8.79 \\
6 & 20.02 & 6.45 & 7.02 & -8.94 \\
7 & 22.80 & 6.27 & 6.72 & -7.09 \\
\hline
\end{tabular}

El algoritmo propuesto, Ec.(1), también resulta útil para obtener de forma inmediata valores de dosis en línea media bajo transmisión parcial, $D_{d / 2}^{\prime}$. Cuando se realizó una comparación entre el valor de dosis calculado y el tomado por la $\mathrm{Cl}$ ubicada en esta posición, se tuvo como resultado una desviación máxima del $8.94 \%$ (Tabla 6), y se encuentra dentro del $\pm 10 \%$ de desviación establecido. Estas desviaciones son del mismo orden que el error experimental. Las principales causas de obtener un porcentaje de desviación alto cuando se usó protección, es debido a que el área proyectada sobre el fantoma, diámetro $=13.16$ $\mathrm{cm}$, protegió necesariamente la región donde se ubicaron los detectores a lo largo del eje central del haz, el resto del fantoma queda expuesto a todo tipo de interacciones y dispersiones producidas por la influencia del haz divergente [1-3].

Se optó por reconstruir una gráfica basada en la relación entre los valores de las dosis $D_{e}, D_{s}$ y $D_{d / 2}$, con la finalidad de analizar la distribución de la dosis a la largo del eje central. Por medio de la gráfica mostrada en la Figura 5 la cual muestra que la relación entre los valores de dosis $D_{d / 2}$ y $D_{e}$ incrementa en forma constante de mayores a menores espesores con respecto a la relación entre los valores de dosis $D_{e}$ y $D_{s}$. Estos datos se ajustan perfectamente a una recta permitiendo ser aplicados en casos reales y obtener valores de la dosis en plano medio a partir de las dosis de entrada y salida, independiente del espesor y edad del paciente [1]

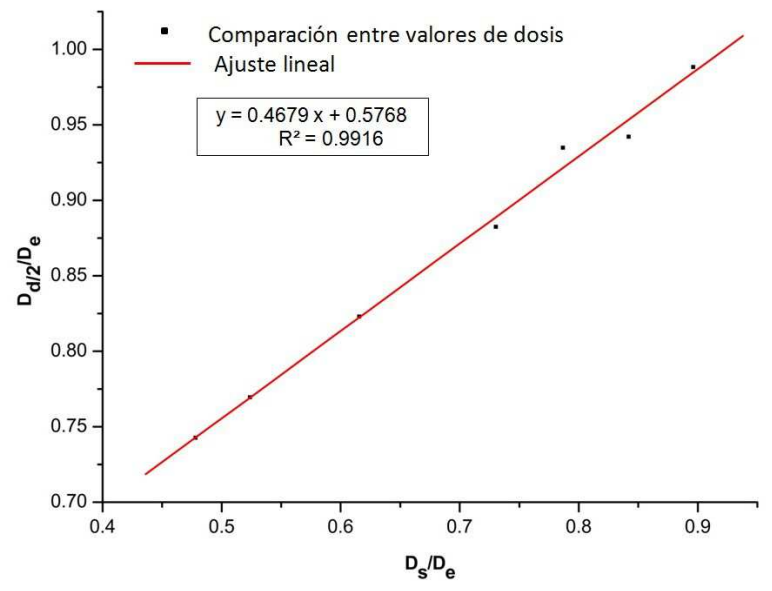

Figura 6: Se muestra $D_{d / 2} / D_{e}$ como una función de $D_{s} / D_{e}$. $D_{d / 2}, D_{e}$ y $D_{s}$ son obtenidos en forma experimental.

De igual forma se graficó la relación entre los valores de dosis $D_{e}^{\prime}, D_{s}^{\prime}$ y $D_{d / 2}^{\prime}$, con la misma finalidad de analizar la distribución de la dosis a la largo del eje central pero esta vez bajo transmisión parcial. La gráfica mostrada en la Figura 6 muestra que la relación entre los valores de dosis $D_{d / 2}^{\prime}$ y $D_{e}^{\prime}$ incrementa de mayores a menores espesores con respecto a la relación entre los valores de dosis $D_{e}^{\prime}$ y $D_{s}^{\prime}$ pero en forma distorsionada debido a los efectos de dispersión y reducción de la tasa de dosis causados por la protección plomada. Estos datos se ajustan a una ecuación polinomial y también pueden utilizarse como referencia para identificar fácilmente valores de dosis en plano medio conociendo las dosis de entrada y salida; independiente del espesor del paciente para casos de protección de órganos críticos [1,2]. 


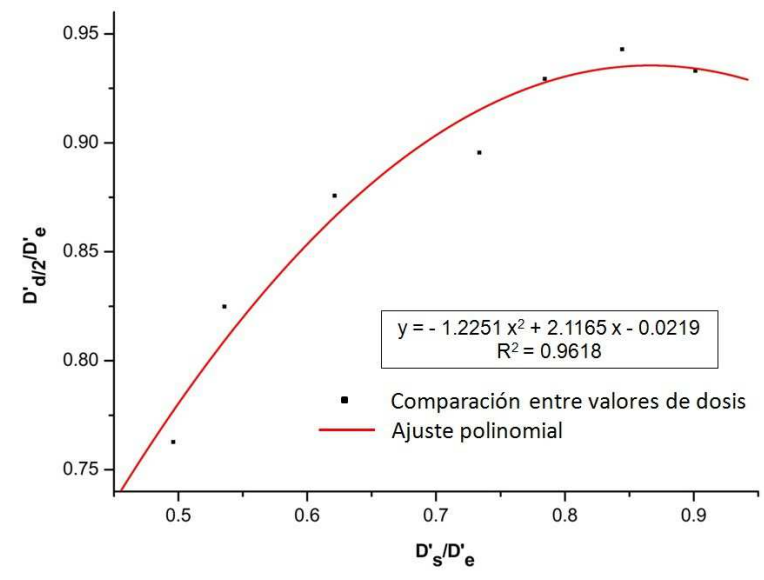

Figura 7: Son mostrados $D_{d / 2}^{\prime} / D_{e}^{\prime}$ como una función de $D_{s}^{\prime} / D_{e}^{\prime} . D_{d / 2}^{\prime}, D_{e}^{\prime}$ y $D_{s}^{\prime}$ son obtenidos en forma experimental, bajo transmisión parcial.

Finalmente teniendo como referencia las curvas específicas, Figuras 5 y 6 , a partir de los valores de las dosis de entrada, de salida y del plano medio, y teniendo el número de UM como referencia, se puede llevar a cabo el tratamiento de ICT requerido [1, 3, -5].

\section{Conclusiones}

Luego de evaluar la curva de PDP vs $d$, Figura 4, se observaron algunos valores dispersos, esto se dió principalmente a que las condiciones dosimétricas difieren de las de una técnica estándar, en cuanto a las profundidades donde se ubica la $\mathrm{Cl}$, el tipo de fantoma, el tamaño de campo y la DFS usados en casos de ICT.

La evaluación de la calidad del haz bajo transmisión parcial mostrado en la curva de $P D P^{\prime}$ vs $d$, Figura 4, no solo se ve afectada por realizarse en condiciones dosimé- tricas diferentes a las usuales sino también al uso de una protección plomada, que afecta negativamente la salida del haz, produciendo mayores efectos de dispersión, que influyen en la disminución de la tasa de dosis.

El algoritmo propuesto en el presente trabajo ha sido utilizado de manera satisfactoria para ambos casos, pudiéndose observar una desviación máxima de $4.58 \%$ cuando no se utilizó protección, resultado que se encuentra dentro del límite establecido; por otro lado cuando se usó protección, la desviación alcanzó un máximo de $8.94 \%$. Las causas que generan este alto porcentaje y una sobreestimación o infraestimación de la dosis es que no se aplicaron los factores de corrección adecuados según la ubicación de los dosímetros, además de una calibración específica que influye en la sensibilidad del dosímetro. Otros efectos que también deben considerarse es la influencia de la divergencia del haz a mayores profundidades, el incremento del tamaño de campo, los factores de atenuación y de dispersión generados por el fantoma y el uso de la protección plomada.

El porcentaje de atenuación $22.72 \%$, supera el estipulado por el protocolo $(20 \%)$, esto conllevó a un aumento en la perturbación del haz, mayores efectos de dispersión y por tanto una reducción de la dosis absorbida por el fantoma, generando mayores desviaciones con respecto a los resultados. Las consecuencias de este exceso requieren de un factor de calibración que dependa del espesor de los discos de plomo en todas las mediciones.

Teniendo como resultado porcentajes de desviación que no superaron el $\pm 10 \%$, se puede optar por el uso de este algoritmo, y por tanto el uso de dosímetros OSL durante la dosimetría in vivo, ya que permite desarrollar un método dosimétrico alternativo, para el cálculo de dosis en plano medio; independiente del espesor y la edad del paciente; que contribuye además a garantizar la calidad del tratamiento.

\section{Referencias}

[1] M. Rivas, N. Jornet, T. Eudaldo, D. Carabante, M. Amor, M. Ginjaume, G. Gómez, F. Sánchez; Radiotherapy Oncology 4951 (1998).

[2] J. Van, J. M. Galvin, G. P. Glasgow, E. B. Podgorsak; A Report of Task Group 29, Radiation Therapy Committee American Association of Physicists in Medicine AAPM Report $N^{o} 17$, pp.4-6, 10-12, 26-38, New York (1986).

[3] K. Bratengeier, R. Müller, K. Rittmann, Whole Body Radiotherapy, DGMP WG 2, Total Body Irradiation Report $N^{o}$ 18, Association of Medical Physics e. V., pp.3-19, Berlín (2003).
[4] E. B. Podgorsak, Radiation Oncology Physics: A Handbook for Teachers and Students: pp. 77, 78, 166196, 264-270, 282, 283, 491, 501, 516, IAEA, Austria (2005).

[5] F. M. Khan, The Physics of Radiation Therapy, pp. 131-132, 179-185, Williams and Wilkins, Second Edition, Philadelphia (1994).

[6] F.H. Attix, Introduction to radiological physics and radiation dosimetry, pp. 26-29, John Wiley and Sons, Inc., Wisconsin (1986).

[7] M. A. Duch, M. Ginjaume, H. Chakkor, X. Ortega, N. Jornet y M. Ribas; Radiotherapy Oncology 47, 31 (1998). 
[8] L. Botter-Jensen, S. W. S. McKeever, A. G. Wintle, Optically Stimulated Luminiscence Dosimetry, pp. 111, 71-79, 101-104, Elsevier Science B. V., Amsterdam (2003).

[9] Colección de Informes Técnicos $N^{\circ} 398$, Determinación de la dosis absorbida en radioterapia con haces externos: Un Código de Práctica Internacional para la dosimetría basada e patrones de dosis absorbida en agua, pp. 55-64, Organismo Internacional de Energía Atómica, OIEA, Viena (2005).

[10] ICRU, Radiation quantities and units. Report 33. International Commission on Radiation Units and Measurements, 7910 Woodmont Ave., Bethesda, MD 20814 (1980). 